Bio - grafía. Escritos sobre la Biología y su Enseñanza. ISSN 2027-1034

Edición Extraordinaria. p.p. 780 - 788

Memorias del IX Encuentro Nacional de Experiencias en Enseñanza de la Biología y la Educación Ambiental. IV Congreso Nacional de Investigación en Enseñanza de la Biología.

\title{
INFLUENCIA DE LA UNIDAD DIDÁCTICA "SUBE LATEMPERATURA" ESTRUCTURADA DESDE EL MODELO DE INVESTIGACIÓN DIRIGIDA, EN LA MODIFICACIÓN DE LOS CONTENIDOS VERBALES SOBRE EL CALENTAMIENTO GLOBAL EN ESTUDIANTES DE GRADO OCTAVO, DEL INSTITUTO TÉCNICO INDUSTRIAL FRANCISCO JOSÉ DE CALDAS, BOGOTÁD.C.
}

\author{
Geraldine Yineth Páez Pérez \\ Jeison Eduardo García Ariza ${ }^{1}$
}

\section{RESUMEN}

El calentamiento global es la teoría que plantea el incremento de las temperaturas del planeta y sus posibles efectos en éste. Por lo anterior, se diseñó, implementó y evaluó la unidad didáctica "Sube la temperatura" para reconocer su influencia en la modificación de los contenidos verbales en 34 estudiantes de grado octavo sobre el calentamiento global, del Instituto Técnico Industrial Francisco José de Caldas, Bogotá D.C. Los instrumentos de recolección de datos fueron dos cuestionarios, uno inicial y otro final, que permitieron reconocer las representaciones de los estudiantes, las cuales se categorizaron en los contenidos verbales (dato-hecho, asociación y concepto) señalados por Pozo (1998). Los resultados iniciales expresan que el $82 \%$ y $18 \%$ de los alumnos se encontraban en la categoría dato-hecho y asociación, respectivamente; mientras que en los finales el $47 \%$, $24 \%$ y $29 \%$ de éstos se ubicaron en las categorías dato-hecho, asociación y concepto, respectivamente. Tales cambios y permanencias pueden deberse a la complejidad de los procesos de comprensión, al desarrollo o no de competencias por los estudiantes y a las dificultades del modelo investigación dirigida. Se concluye que este trabajo generó algunos cambios en los contenidos verbales de los estudiantes.

PALABRAS CLAVE: Categorías verbales, investigación dirigida, calentamiento global.

\section{ABSTRACT}

Global warming is the theory that says about the increases planet's temperature and its possible effects. Therefore, the aim of present work was to recognize the influence of the didactic unit "Sube la temperature" in the modification of the verbal contents in 34 students of eighth grade about global warming, of the Industrial Technical Institute Francisco José de Caldas, Bogota D.C. The instruments to data collection were two questionnaires, one initial and other final, which allowed be recognize the student's representations that were categorized in the verbal contents (data-fact, association and concept) taken of Pozo (1998). The initial results showed that $82 \%$ and $18 \%$ of students were placed in the data-

\footnotetext{
${ }^{1}$ Universidad Distrital Francisco José de Caldas, Facultad de Ciencias y Educación. Estudiantes de licenciatura en Biología. Correos electrónicos: yinethpp@hotmail.com/jeisonpsique@hotmail.com
} 


\title{
Bio - grafía. Escritos sobre la Biología y su Enseñanza. ISSN 2027-1034
}

\section{Edición Extraordinaria. p.p. 780 - 788}

\author{
Memorias del IX Encuentro Nacional de Experiencias en Enseñanza de la Biología y la \\ Educación Ambiental. IV Congreso Nacional de Investigación en Enseñanza de la \\ Biología.
}

fact and association category, respectively; while in the end results, $47 \%, 24 \%$ and $29 \%$ of the students were placed in the data-fact, association and concept categories, respectively. Such changes and permanencies were explained by the complexity of comprehension processes, the development of the competences and the difficulties of the model directed research. It is concluded that this work generated some changes in the verbal contents of the students.

KEY WORDS: Verbal categories, directed research, global warming.

\section{INTRODUCCIÓN}

El incremento del valor medio de la temperatura en la atmósfera terrestre y océanos, principalmente desde el comienzo de la revolución industrial, permitió el desarrollo de la teoría del calentamiento global que sostiene el aumento de las temperaturas del planeta (Duque, 2011). Así, para el rango de $1901-2012$ se muestra un ascenso de $0.85^{\circ} \mathrm{C}$, lo cual implica una alteración del ciclo del agua, un cambio en el nivel del mar, la extinción de especies animales, entre otros, planteándose una relación con acciones que aumentan los gases de efecto invernadero (Stocke, Dahe, \& Plattner, 2013).

Como estrategia para afrontar esta situación, el presente trabajo diseñó, implementó y evaluó una Unidad Didáctica sobre el calentamiento global, entendida ésta como la planeación que hace el docente de sus clases y que incluye un análisis científico, análisis didáctico, selección de objetivos, selección de estrategias didácticas y selección de estrategias de evaluación (Sánchez \& Valcárcel, 1993).

El modelo de investigación dirigida, en el marco de las estrategias didácticas, se adoptó porque reconoce las contribuciones de la historia y epistemología de la ciencia, afirmando que el estudiante debe situarse en un ambiente próximo a la labor cotidiana de un científico, entendiendo ésta como un proceso complejo que implica la presencia de un problema, la construcción de hipótesis, el diseño de formas de confrontación, la experimentación, la obtención y análisis de resultados, la comunicación de éstos, entre otros (Gil, 1993).

El estudiante a partir de lo anterior, puede verse desde dos aspectos: sus competencias y sus componentes. Las competencias como la habilidad del estudiante que refleja la forma cómo razona y explica el mundo natural y social, a través de la interpretación de conceptos científicos (Chona, y otros, 2006), las cuales pueden desarrollarse con el enfoque de investigación dirigida (Fonseca, 2008); los componentes, corresponden a lo conceptual, metodológico y actitudinal, elementos que deben trabajarse bajo la atenta dirección del docente (Pozo \& Gómez, 1998).

El análisis del elemento conceptual, o contenido verbal, es importante porque es el punto de referencia para el desarrollo del currículo en ciencias y adicionalmente en él puede reconocerse las relaciones de significación que el estudiante ha elaborado para describir y comprender un fenómeno. Este puede dividirse en tres categorías: dato-hecho, concepto y principio (Pozo \& Gómez, 1998). 
Bio - grafía. Escritos sobre la Biología y su Enseñanza. ISSN 2027-1034

Edición Extraordinaria. p.p. 780 - 788

Memorias del IX Encuentro Nacional de Experiencias en Enseñanza de la Biología y la

Educación Ambiental. IV Congreso Nacional de Investigación en Enseñanza de la

Biología.

A partir de todo lo anterior, el presente trabajo tuvo como objetivo reconocer la influencia de la Unidad Didáctica "Sube la temperatura" estructurada desde el modelo de Investigación dirigida, en la modificación de los contenidos verbales sobre el calentamiento global en estudiantes de grado octavo, del Instituto Técnico Industrial Francisco José de Caldas, Bogotá D.C., bajo el marco de la Práctica profesional III de la carrera Licenciatura en Biología de la Universidad Distrital F.J.C.

\section{METODOLOGÍA}

El presente trabajo se desarrolló bajo el paradigma Interpretativo que se enfoca en el estudio de los símbolos, planteando interpretaciones y significados de las acciones humanas y de la vida social, lo cual, implica que la realidad se construye socialmente, a partir de los significados específicos de un contexto (Montenegro \& López, 2006).

El trabajo se desarrolló en el Instituto Técnico Industrial Francisco José de Caldas, ubicado en la localidad de Engativá, Bogotá D.C., con 34 estudiantes de grado octavo, jornada tarde, de los cuales 21 eran hombres y 13 mujeres, entre los 12 y 17 años de edad. Se desarrollaron diez (10) sesiones de clase en relación al calentamiento global, cada una enfocada a desarrollar una o varias competencias (ver Tabla 1) desde la clasificación planteada por Chona y otros (2006).

\begin{tabular}{|c|c|c|c|}
\hline No. & TEMA & ACTIVIDAD & COMPETENCIA \\
\hline 1 & $\begin{array}{l}\text { Calentamiento } \\
\text { global }\end{array}$ & $\begin{array}{l}\text { Implementación de cuestionario } \\
\text { de ideas previas }\end{array}$ & Proponer posibles explicaciones. \\
\hline 2 & $\begin{array}{l}\text { Cambio } \\
\text { climático }\end{array}$ & Planteamiento de la problemática & $\begin{array}{l}\text { Proponer o establecer los } \\
\text { procedimientos para abordar } \\
\text { problemas. }\end{array}$ \\
\hline 3 & $\begin{array}{l}\text { Causas del } \\
\text { calentamiento }\end{array}$ & $\begin{array}{l}{ }^{*} \text { Desarrollo de práctica sobre la } \\
\text { descomposición de la basura. Se } \\
\text { usó botella y bolsa plástica, } \\
\text { pitillos y cáscara de naranja y } \\
\text { banano. } \\
{ }^{*} \text { Creación de diario para el } \\
\text { registro semanal de los } \\
\text { elementos nombrados }\end{array}$ & $\begin{array}{l}\text { *Identificar los diseños experimentales } \\
\text { pertinentes para contrastar hipótesis. } \\
\text { *Proponer posibles explicaciones. } \\
\text { *Predecir los resultados del proceso. }\end{array}$ \\
\hline 4 & Ciclo de $\mathrm{O}_{2}$ & $\begin{array}{l}\text { *Discusión grupal sobre la } \\
\text { participación del oxígeno en } \\
\text { procesos biológicos. }\end{array}$ & Proponer posibles explicaciones. \\
\hline
\end{tabular}


Bio - grafía. Escritos sobre la Biología y su Enseñanza. ISSN 2027-1034

Edición Extraordinaria. p.p. 780 - 788

Memorias del IX Encuentro Nacional de Experiencias en Enseñanza de la Biología y la Educación Ambiental. IV Congreso Nacional de Investigación en Enseñanza de la Biología.

\begin{tabular}{|c|c|c|c|}
\hline 5 & Líquenes & $\begin{array}{l}\text { "Discusión sobre su lugar en el } \\
\text { ciclo del oxígeno. Se trabajó con: } \\
\text { Usnea sp., Sticta sp., Ramalina } \\
\text { sp., Telochistes sp., Parmotrema } \\
\text { sp., y líquenes foliosos. }\end{array}$ & $\begin{array}{l}\text { *Proponer posibles explicaciones. } \\
\text { *Generalizar a partir de las } \\
\text { observaciones. }\end{array}$ \\
\hline 6 & $\begin{array}{l}\text { Ciclo del } \\
\text { carbono }\end{array}$ & $\begin{array}{l}\text { *Discusión grupal de los procesos } \\
\text { de respiración y fotosíntesis de } \\
\text { los líquenes. } \\
\text { *Discusión grupal de las acciones } \\
\text { humanas que afectan tanto el } \\
\text { ciclo del oxígeno como el del } \\
\text { carbono. }\end{array}$ & $\begin{array}{l}{ }^{*} \text { Capacidad para observar y describir } \\
\text { objetos, eventos o fenómenos. } \\
\text { *Tomar decisiones, en la selección de } \\
\text { información, datos o procedimientos. } \\
{ }^{*} \text { Construir y reconstruir significados a } \\
\text { partir de la información consultada. } \\
\text { ”Ser coherente entre su pensamiento y } \\
\text { acción. }\end{array}$ \\
\hline 7. & Basura & $\begin{array}{l}\text { "Elaboración de una línea de } \\
\text { tiempo de la descomposición de } \\
\text { diferentes elementos. } \\
\text { *Discusión grupal de la relación } \\
\text { de la basura con el ciclo del } \\
\text { oxígeno y carbono. }\end{array}$ & $\begin{array}{l}\text { “Proponer posibles explicaciones. } \\
\text { *Desarrollo de pensamiento causal. } \\
\text { *Utilizar pensamiento crítico y creativo } \\
\text { en la solución de problemas. } \\
\text { *Formular y contrastar hipótesis. }\end{array}$ \\
\hline 8 & Combustión & $\begin{array}{l}\text { "Práctica sobre la combustión, en } \\
\text { la cual se analiza el papel del } \\
\text { oxígeno en este proceso. Se usó } \\
\text { velas de diferentes tamaños y } \\
\text { grosor y vasos de vidrio. }\end{array}$ & $\begin{array}{l}\text { "Proponer posibles explicaciones } \\
\text { *Desarrollo de pensamiento causal. } \\
\text { *Utilizar pensamiento crítico y creativo } \\
\text { en la solución de problemas. } \\
\text { *Formular y contrastar hipótesis. }\end{array}$ \\
\hline 9 & $\begin{array}{l}\text { Calentamiento } \\
\text { global }\end{array}$ & $\begin{array}{l}{ }^{*} \text { Construcción } \text { grupal de red } \\
\text { conceptual que relacione todos } \\
\text { los temas y conceptos vistos } \\
\text { durante el proceso. }\end{array}$ & $\begin{array}{l}\text { "Proponer posibles explicaciones. } \\
\text { *Desarrollo de pensamiento causal. } \\
\text { *Utilizar pensamiento crítico y creativo } \\
\text { en la solución de problemas. }\end{array}$ \\
\hline 10 & $\begin{array}{l}\text { Cuestionario } \\
\text { final }\end{array}$ & $\begin{array}{l}\text { * Implementación } \\
\text { cuestionario final. }\end{array}$ & \\
\hline
\end{tabular}

La metodología de obtención de datos consistió en el diseño e implementación de dos cuestionarios para el reconocimiento de las representaciones de los estudiantes sobre el calentamiento global; así, un cuestionario se aplicó al inicio y otro al final del proceso. 
Bio - grafía. Escritos sobre la Biología y su Enseñanza. ISSN 2027-1034

Edición Extraordinaria. p.p. 780 - 788

Memorias del IX Encuentro Nacional de Experiencias en Enseñanza de la Biología y la

Educación Ambiental. IV Congreso Nacional de Investigación en Enseñanza de la Biología.

Los cuestionarios compartían la sección de escribir y dibujar sobre las características, causas y consecuencias del calentamiento global y se diferenciaban en que el cuestionario inicial incluía una encuesta sobre acciones que promueven el calentamiento global.

Las respuestas de cada estudiante, tanto iniciales como finales, fueron ubicados en las categorías verbales señaladas por Pozo (1998), pero con una adaptación operativa, tal como se muestra a continuación: dato-hecho corresponde a enunciación memorística; asociación, al proceso de relación de datos y hechos, pero con algunas incoherencias, es decir, con un vínculo no adecuado de las afirmaciones planteadas; concepto, a la asociación coherente de datos y hechos que permiten explicar adecuadamente un fenómeno y principio, como el reconocimiento abstracto de los conceptos estructurales que hacen parte de la teoría.

\section{RESULTADOS}

El cuestionario inicial permitió reconocer que respecto a las siguientes actividades: ducharse con agua caliente (DCC), montar bicicleta (MB), cargar el celular (CC), reciclar (RRR), usar internet (UI), extracción de petróleo (EP), manejo de automóvil (MA), usar plancha secador (PL/SEC) y talar árboles (TA), el 100\%, 97\% y $88 \%$ de los estudiantes considera que manejar automóvil, la tala de árboles y la extracción de petróleo, respectivamente, promueve el calentamiento global; mientras que acciones cotidianas como ducharse con agua caliente, cargar el celular y utilizar plancha/secador tienen un porcentaje de $50 \%$, 73\% y $44 \%$, respectivamente (ver Gráfica $2 \mathrm{~A}$ ).

La categorización y sistematización de los cuestionarios iniciales permitió reconocer que el $82 \%$ de los estudiantes se encontraba en la categoría de dato-hecho, el $18 \%$ en asociación y $0 \%$ en concepto (ver Gráfica $2 \mathrm{~B}$ ). A continuación, se presentan algunos fragmentos de los textos de los cuestionarios de los estudiantes, para ejemplificar cada una de dichas categorías:

"El calentamiento es cuando la capa de ozono se debilita por todos los gases que se producen en la tierra y con el efecto invernadero" JD

"El calentamiento global es cuando los rayos del sol recalientan el planeta Tierra, y estos rayos nos afectan a nosotros". LS

"El calentamiento global es el daño o destrozo de la capa de ozono por botar basura y esto produce que el sol caliente más y haga más calor; por eso, se derriten los polos y se secan los ríos" DT

"Es el daño de la atmósfera que provoca que la temperatura suba, ya que los rayos solares entran a la tierra y la contaminación no deja que estos salgan, y (Asociación) esto sube la temperatura" SY

Los cuestionarios finales permitieron reconocer que, luego de las diez sesiones desarrolladas, el $47 \%, 24 \%$ y $29 \%$ de los estudiantes se encontraba en el contenido 


\title{
Bio - grafía. Escritos sobre la Biología y su Enseñanza. ISSN 2027-1034
}

\section{Edición Extraordinaria. p.p. 780 - 788}

\author{
Memorias del IX Encuentro Nacional de Experiencias en Enseñanza de la Biología y la \\ Educación Ambiental. IV Congreso Nacional de Investigación en Enseñanza de la \\ Biología.
}

verbal de dato-hecho, asociación y concepto, respectivamente. Lo cual, permite observar una disminución de los estudiantes que se ubicaban en la categoría dato-hecho y el progreso de otros estudiantes a la categoría de concepto (ver Gráfica C). A continuación, se presentan algunos fragmentos de los cuestionarios finales de los estudiantes:

"[...] El calentamiento global tiene que ver con los estados del clima y la capa de ozono va cambiando, va cubriendo el planeta para que este pueda verse beneficiado [...]" SB

"[...] El calentamiento global puede aumentar por varios factores que afectan el efecto invernadero tal como el daño que se le hace a la capa de ozono, [...] (Asociación) por diferentes tipos de gases que provocan que los rayos solares entren con más frecuencia a la tierra [...]" KR

"[...] El calentamiento global está causado por varias razones, una de ellas es la quema de basura y la expulsión de $\mathrm{CO}_{2}$ que produce que varios gases se (Concepto) acumulen en la atmosfera y que el efecto invernadero se vea intensificado logrando que los rayos ultravioleta se queden más tiempo en la tierra [...]" JD

(Dato-Hecho)

Las categorías verbales de los cuestionarios inicial y final tenían más similitudes que diferencias en relación a su tema. Así, las semejanzas en la categoría dato incluía a los rayos solares, capa de ozono, clima, gases, efecto invernadero, lluvia ácida, ciclo del agua, dióxido de carbono y atmósfera; mientras que, como diferencia sólo al inicio se enunció el daño a la atmósfera (ver Gráfica 2D). La categoría hecho incluía al aumento de la temperatura, derretimiento de los polos, congelamiento de la tierra, sequía, inundaciones, contaminación, uso de aerosoles, basura, sobreexplotación, gasto de energía, tala de árboles, industrialización y extracción de petróleo, sin presentar diferencias (ver Gráfica 2E) y la categoría asociación incluía a datos y hechos ya mencionados como: el aumento de la temperatura, contaminación, combustión, basura, derretimiento de los polos, sequía, sobreexplotación, ecosistemas, industrialización, tala de árboles, capa de ozono, rayos solares y dióxido de carbono, incluyendo a la industrialización y el daño en la atmósfera sólo el cuestionario inicial (ver Gráfica 2G).

\section{DISCUSIÓN DE RESULTADOS}

La comparación de las categorías verbales de los cuestionarios iniciales y finales, permitió reconocer cambios en los estudiantes, ya que inicialmente el $82 \%$ de ellos se ubicaban en la categoría dato-hecho y posteriormente sólo el 47\%; la categoría asociación se amplió de un $18 \%$ a un $24 \%$; la categoría concepto, que estuvo ausente en los cuestionarios iniciales, tuvo lugar con una representación del $29 \%$ de los alumnos en el cuestionario final y la categoría principio no tuvo participación en ambos cuestionarios. La permanencia y cambio de las categorías verbales en los estudiantes puede entenderse porque los procesos cognitivos que implica la comprensión de algún fenómeno natural son altamente complejos y progresivos (Pozo \& Gómez, 1998); lo cual implica, que los estudiantes 
Bio - grafía. Escritos sobre la Biología y su Enseñanza. ISSN 2027-1034

Edición Extraordinaria. p.p. 780 - 788

Memorias del IX Encuentro Nacional de Experiencias en Enseñanza de la Biología y la

Educación Ambiental. IV Congreso Nacional de Investigación en Enseñanza de la Biología.

desde sus representaciones iniciales estaban construyendo paulatinamente significaciones sobre el calentamiento global.

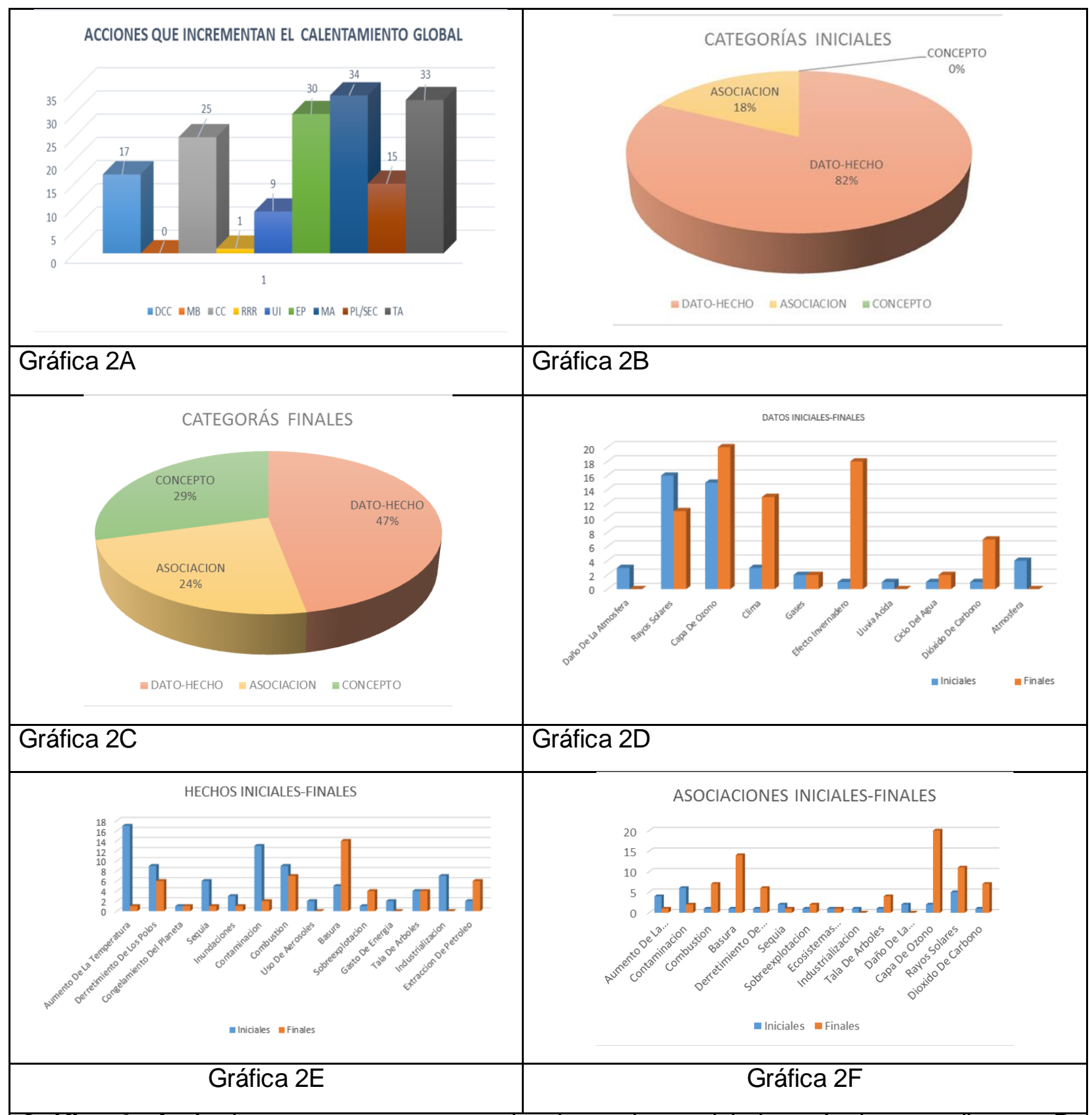

Gráfica 2. A. Acciones que promueven el calentamiento global según los estudiantes; B. Categorías verbales de los cuestionarios iniciales; C. Categorías verbales de los cuestionarios 
Bio - grafía. Escritos sobre la Biología y su Enseñanza. ISSN 2027-1034

Edición Extraordinaria. p.p. 780 - 788

Memorias del IX Encuentro Nacional de Experiencias en Enseñanza de la Biología y la

Educación Ambiental. IV Congreso Nacional de Investigación en Enseñanza de la Biología.

\begin{abstract}
finales; D. Datos enunciados al inicio y al final; E. Hechos enunciados al inicio y al final $\mathbf{F}$. Asociaciones planteadas en el cuestionario inicial y final.

El cambio y mantenimiento de las categorías verbales, adicionalmente, puede relacionarse con el trabajo de las competencias señaladas en el apartado de la metodología, ya que el desarrollo o no de ciertas habilidades por parte del estudiante influye en la construcción de su modelo mental sobre el fenómeno (Espinet \& Ramos, 2012); es decir, el no desarrollo de ciertas competencias por parte del alumno puede ayudar a la comprensión del por qué permaneció en la categoría dato o hecho.

El modelo de investigación dirigida al situar al estudiante en un ambiente semejante al del científico, plantea una serie de dificultades prácticas relacionadas con las preocupaciones de los estudiantes respecto a la de los científicos (Pozo \& Gómez, 1998), lo cual se constató en el desarrollo de las sesiones cuando las actitudes de todos los estudiantes no eran semejantes en relación a los elementos de la labor científica.
\end{abstract}

\title{
CONCLUSIÓN
}

El análisis de los cuestionario iniciales y finales de 34 estudiantes de grado octavo, tras la implementación de la unidad didáctica "Sube la temperatura" estructurada desde el modelo de investigación dirigida, permite reconocer cambios en sus categorías verbales disminuyendo el porcentaje de alumnos que estaban en la categoría dato-hecho y ampliándose el porcentaje de la categoría concepto; lo cual, debe interpretarse desde las dificultades que plantea la investigación dirigida y las competencias científicas.

\section{BIBLIOGRAFÍA}

Chona, G., Judith, A., Martínez, S., Ibañez, X., Pedraza, M., \& Fonseca, G. (2006). ¿Qué competencias científicas promovemos en el aula? Tecné, Episteme y Didaxis, 6279.

Duque, G. (5 de Junio de 2011). Calentamiento global en Colombia. Bogotá D.C., Colombia.

Espinet, M., \& Ramos, S. (2012). Identificar y explicar los errores en las actividades experimentales una estrategia para desarrollar competencias científicas. En E. Badillo, L. García, A. Marbá, \& B. Mauro, Desarrollo de competencias en la clase de ciencias y matemáticas (págs. 141-162). Mérida: Universidad de los Andes.

Fonseca, G. (Julio de 2008). La implementación del enfoque didáctico de investigación dirigida y el desarrollo de competencias cientificas en un grupo de estudiantes de educación básica. Obtenido de El portal de los académicos: http://academicos.iems.edu.m $\mathrm{x} /$ cired/docs $/ \mathrm{tg} / \mathrm{macroacademiaquimica/Formacion \%}$ $20 \mathrm{\%} 20$ evaluacion\%20de\%20competencias\%20cinetificas_FonsecaAmaya.pdf 
Bio - grafía. Escritos sobre la Biología y su Enseñanza. ISSN 2027-1034

Edición Extraordinaria. p.p. $780-788$

Memorias del IX Encuentro Nacional de Experiencias en Enseñanza de la Biología y la Educación Ambiental. IV Congreso Nacional de Investigación en Enseñanza de la Biología.

Gil, D. (1993). Contribución de la historia y de la filosofía de las ciencias al desarrollo de un modelo de enseñanza/aprendizaje como investigación. Enseñanza de las ciencias, 197-212.

Montenegro, M., \& López, L. (2006). Interrelación de la investigación y la docencia en el programa de Derecho. Resultado de un proceso de investigativo. Bogotá D.C.: Universidad Cooperativa de Colombia.

Pozo, J., \& Gómez, M. (1998). Aprender y enseñar ciencia: del conocimiento cotidiano al conocimiento científico. Madrid: Ediciones Morata.

Sánchez, G., \& Valcárcel, M. (1993). Diseño de unidades didácticas en el área de ciencias experimentales. Enseñanza de las ciencias, 33-44.

Stocke, T., Dahe, Q., \& Plattner, G.-K. (2013). Contribución del Grupo de trabajo I al Quinto Informe de Evaluación del Grupo Intergubernamental de Expertos sobre el Cambio Climático. Cambridge University Press, Cambridge, Reino Unido y Nueva York: IPCC. 